\title{
3DVEM SOFTWARE MODULES FOR EFFICIENT MANAGEMENT OF POINT CLOUDS AND PHOTOREALISTIC 3D MODELS
}

\author{
S. Fabado, A. E. Seguí, M. Cabrelles, S. Navarro, D. García-De-San-Miguel, J. L. Lerma*
}

\begin{abstract}
Photogrammetry \& Laser Scanner Research Group (GIFLE), Department of Cartographic Engineering, Geodesy and Photogrammetry, Universitat Politècnica de València, $C^{o}$ de Vera, s/n, Building 7i, 46022 Valencia, Spain - (sanfasal, ansegil, micablo, sannata, dagarsa4, jllerma)@upv.es
\end{abstract}

KEY WORDS: Cultural Heritage, Management, Visualization, Point Cloud, DEM/DTM, Lidar, Registration, Animation

\begin{abstract}
:
Cultural heritage managers in general and information users in particular are not usually used to deal with high-technological hardware and software. On the contrary, information providers of metric surveys are most of the times applying latest developments for real-life conservation and restoration projects. This paper addresses the software issue of handling and managing either 3D point clouds or (photorealistic) 3D models to bridge the gap between information users and information providers as regards the management of information which users and providers share as a tool for decision-making, analysis, visualization and management. There are not many viewers specifically designed to handle, manage and create easily animations of architectural and/or archaeological 3D objects, monuments and sites, among others. 3DVEM - 3D Viewer, Editor \& Meter software will be introduced to the scientific community, as well as 3DVEM - Live and 3DVEM - Register. The advantages of managing projects with both sets of data, 3D point cloud and photorealistic 3D models, will be introduced. Different visualizations of true documentation projects in the fields of architecture, archaeology and industry will be presented. Emphasis will be driven to highlight the features of new userfriendly software to manage virtual projects. Furthermore, the easiness of creating controlled interactive animations (both walkthrough and fly-through) by the user either on-the-fly or as a traditional movie file will be demonstrated through 3DVEM Live.
\end{abstract}

\section{INTRODUCTION}

There is a large variety of active and passive optical 3D measuring systems available in the market (Blais, 2004) to yield fast and accurate point clouds with either intensity or colour values. Similarly, there is a wide variety of computer vision, photogrammetry and laser scanning software that handles 3D point clouds and models. However, there are not many viewers specifically designed to handle, manage and disseminate easily architectural and/or archaeological 3D objects, monuments and sites.

Information users (i.e. conservation experts specialized in project management, maintenance, monitoring, inventory, conservation and research) and information providers (i.e. heritage recorders specialized in photography, photogrammetry, surveying, GIS, 3D modelling, geophysics, remote sensing...) as defined in Letellier et al. (2007) require fast, intuitive and easy to use software to explore in depth large 3D point clouds, complex 3D models and accurate photorealistic 3D models.

One of the great advantages of having an accurate, high resolution 3D model of an object is the ability to manage, view, analyze, monitor and particularize it to extract relevant information for your needs whenever requested at your own convenience. The number of times that $3 \mathrm{D}$ data coming from third party bodies ends up after its formal reception is unfortunately limitless. No matter the heritage project, the team, the resources used to bring in datasets, the data format, there is a need to bridge the gap between information users and information providers. There are exceptions to the previous statement as far as multidisciplinary teams work closely together, or high specialized experts are able to do both.

Besides management, dissemination of 3D data based on users' needs from original sources is uncommon. The same 3D point clouds, 3D models and photorealistic 3D models should be differently widespread based not only on the purpose but on the audience.

In 3D computer graphics, there exists a wide range of software products aimed to create, render and animate 3D data. The products have different features such as user's mission, industrial sector, cost, license, mono, multi or closed-based functionality.

On the one hand, two leading 3D computer graphics software are Autodesk 3DS Max (Autodesk Inc., 2013a) and Autodesk Maya (Autodesk Inc., 2013b). They are proprietary software designed to make 3D models, animations and rendering, commonly used by videogame developers, film studios and architecture teams. On the other hand, Blender (Blender Foundation, 2012) is an open-source software, comparable to the previously mentioned software in terms of features and functionalities. It inherits the advantages and disadvantages of the open-source philosophy: free license agreement and open development. However, support should not be underestimated and most of the times proprietary products are more supportive. MeshLab (MeshLab, 2012) is another open-source software used to edit and process point clouds and 3D models. It provides a set of tools for editing, cleaning, inspection, processing and conversion between different formats (Cignoni

\footnotetext{
* Corresponding author.
} 
et al., 2008). However, it does neither support modelling new geometries nor making animations.

This article presents three powerful low cost software modules developed by the Photogrammetry \& Laser Scanning Research Group (GIFLE) in the Department of Cartographic Engineering, Geodesy and Photogrammetry, Polytechnic University of Valencia (Spain): 3DVEM - 3D Viewer, Editor \& Meter; 3DVEM - Live and 3DVEM - Register. The first two modules target both information users and information providers to manage and handle point clouds, 3D models and photorealistic 3D models, while the latter is specially design keeping in mind information provider's needs as regards registration (also known as alignment/orientation) of both point clouds and 3D models.

This paper is structured as follows. Sections 2, 3 and 4 deal the existing 3DVEM software modules. In particular, Section 2 starts with the free core proprietary software 3DVEM - 3D Viewer, Editor \& Meter. Section 3 follows with the 3DVEM Register software and explores its capability as an import/export registration toolbox. Section 4 reviews 3DVEM - Live software as an easy to use multimedia animation module to disseminate research, surveys and 3D documentation projects to the society. True photogrammetry and laser scanning documentation projects will be used to display part of the potential of the existing software modules. Finally Section 5 draws some conclusions.

\section{3DVEM - 3D VIEWER, EDITOR \& METER SOFTWARE TO MANAGE POINT CLOUDS AND PHOTOREALISTIC 3D MODELS}

3DVEM - 3D Viewer, Editor \& Meter is the core of a series of modules that allows users an interactive mean to explore, analyze, tag and measure $3 \mathrm{D}$ point clouds, conventional $3 \mathrm{D}$ models and photorealistic 3D models. 3DVEM - 3D Viewer, Editor \& Meter runs on Windows operating system.

3DVEM - 3D Viewer, Editor \& Meter provides users an intuitive, interactive and easy to use $3 \mathrm{D}$ data interface that allows the efficient management of both point clouds and 3D models. Data can be obtained by different means: simple measurements, photogrammetry, computer vision, robotics, computer graphics, laser scanning, etc. Handle of datasets is independent of the way data was acquired: aerial solutions, terrestrial, underwater or underground. Similarly, it is independent of the processing workflow undertaken by the datasets.

Figure 1 illustrates a photorealistic 3D model imported into 3DVEM - 3D Viewer, Editor \& Meter. Cantos de Visera II is a rock art shelter full of paintings on Arabí Mountain (Yecla, Spain). The photorealistic 3D model was achieved by the authors after integrating image-based and range-based data (Lerma and Cabrelles, 2013; Lerma et al., 2010). All the processing was undertaken by the in-house FOTOGIFLE software. Further information and related research about this rock art site can be found in (Ruíz Molina, 2013).

Unlike other software tools on the market, this solution responds to the growing demand for 3D documentation and multidisciplinary data interchange. Experts can make their own notes, measurements, and/or analysis of the uprisings. 3DVEM - 3D Viewer, Editor \& Meter does not only display 3D data but also allows users to perform measurement and custom dimension to suit their needs (Figure 2).

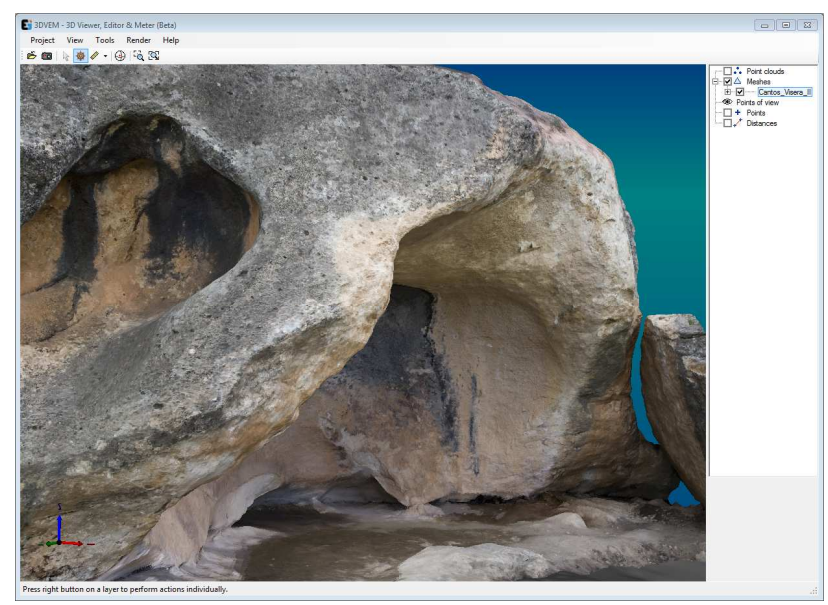

Figure 1. Main window of 3DVEM - Viewer, Editor \& Meter. Visualization of the rock art shelter Cantos de Visera II photorealistic 3D model

The tree structure on the right-hand side of the main window (Figs. 1 and 2) shows all the loaded objects such as point clouds, meshes, points of view, points and polylines. The objects can be either shown or hidden to the user's convenience. In addition, 3DVEM - Viewer, Editor \& Meter provides different ways of rendering the information so that it can be accommodated to the final customer's needs.

Some additional features available in 3DVEM - 3D Viewer, Editor \& Meter software are:

- Import in different formats, clouds of points (ASC, TXT, PTS, PTX) and 3D models (OBJ and VRML).

- Select and save different viewpoints.

- Study the virtual world either in perspective or in orthographic view.

- Displays point clouds in RGB, intensity or single colour point cloud.

- Modify the shape and size of the point.

- $\quad$ Record and customize 3D measurements.

- $\quad$ Apply different renderings to models.

- $\quad$ Change the type of lighting to emphasize the 3D models.

Currently the beta version of the program can be free downloaded from GIFLE (2013). 


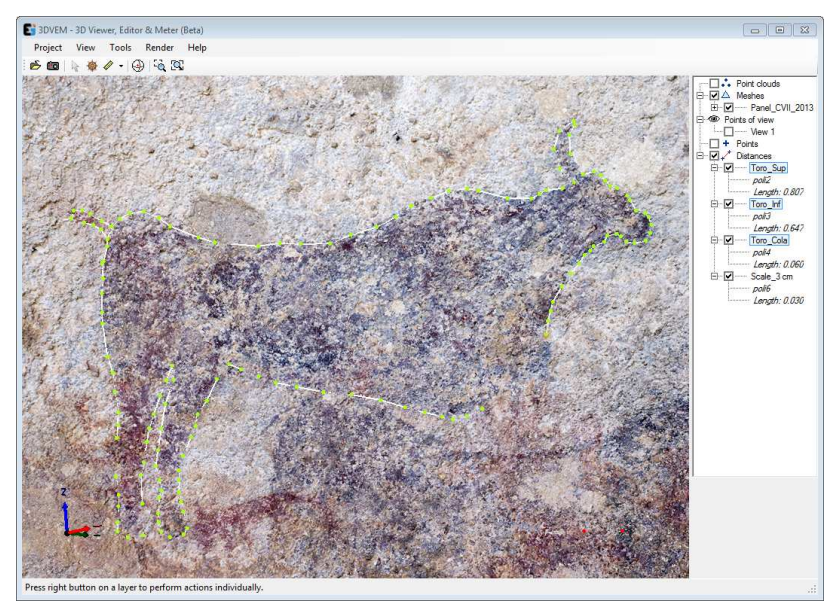

(a)

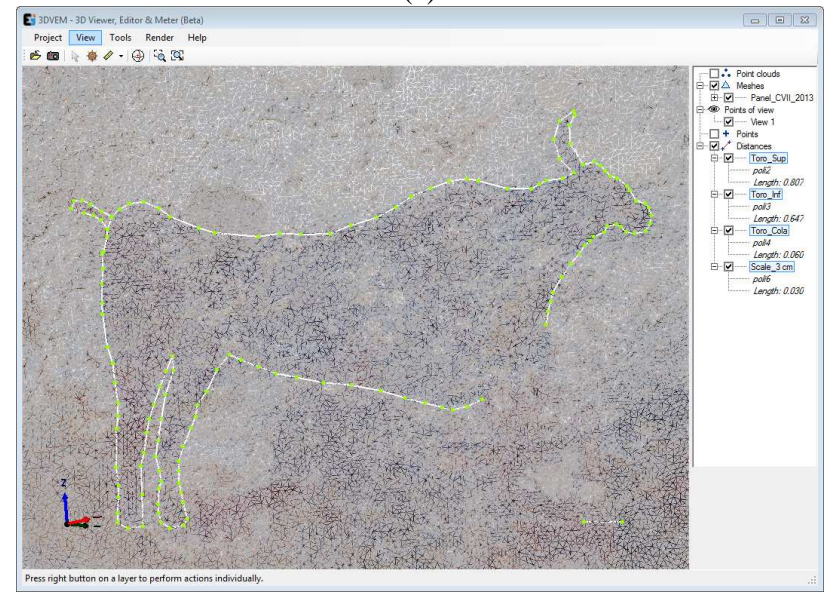

(b)

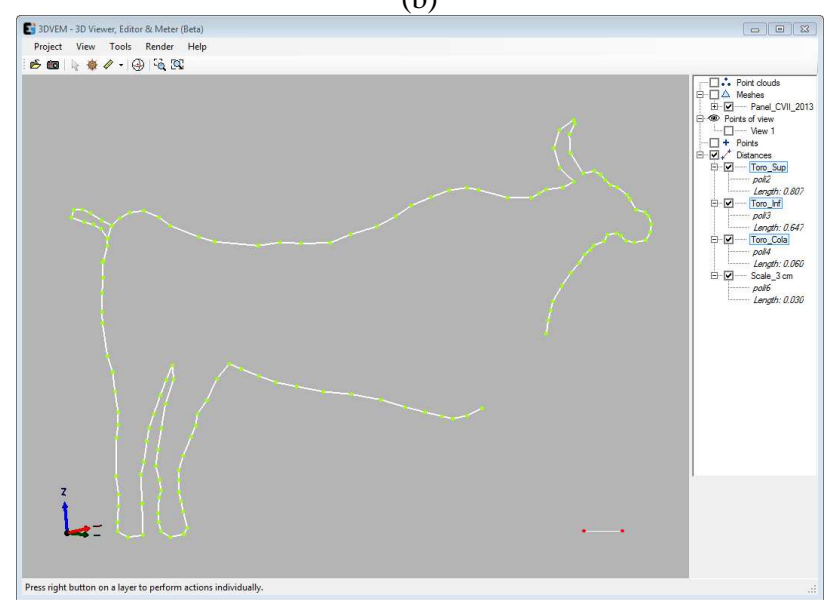

(c)

Figure 2. Viewpoint selection and 3D drawings/measurements from a single rock art motif from Cantos de Visera II: a) photorealistic 3D model; b) wireframe model; c) undertaken drawings only

\section{3DVEM - REGISTER SOFTWARE TO ALIGN, ORIENT OR REGISTER POINT CLOUDS AND/OR 3D MODELS}

Registration of laser scanning data, also known as alignment or orientation relative is used to transform multiple 3D Cartesian coordinate systems captured by laser scanners into one common reference system.
The registration process is essential in any job that requires more than one scan position. A good fit ensures that the remaining operations (filtering, sections, elevations, triangulation, smoothing, etc.) are integrated better into a single reference system. In this regard, Bryan et al. (2003) established the following requirements for scanned data control by resection calculations:

- At least four accurate control points should be suitably distributed in space.

- The residuals of the registration process and geometric precision of the estimated parameters should be equal to or better than the required accuracy for the final point cloud.

3DVEM - Register (Fig. 3) is a low cost, easy to use and reliable alternative to manufacturer software packages. It solves the indirect registration between point clouds and/or 3D models through a 3D rigid transformation. To carry out the registration it is necessary to know the coordinates of the centres of the targets/spheres/natural points measured for each setup, that can be imported through ASCII files. The numbering of the entities is in principle random: 3DVEM - Register sets automatically matches solves the indirect registration either by least squares or robust estimators, depending on the option selected by the user. Additionally, the interface is friendly and intuitive, and provides sufficient statistical data to accept or recalculate the best adjustment solution.

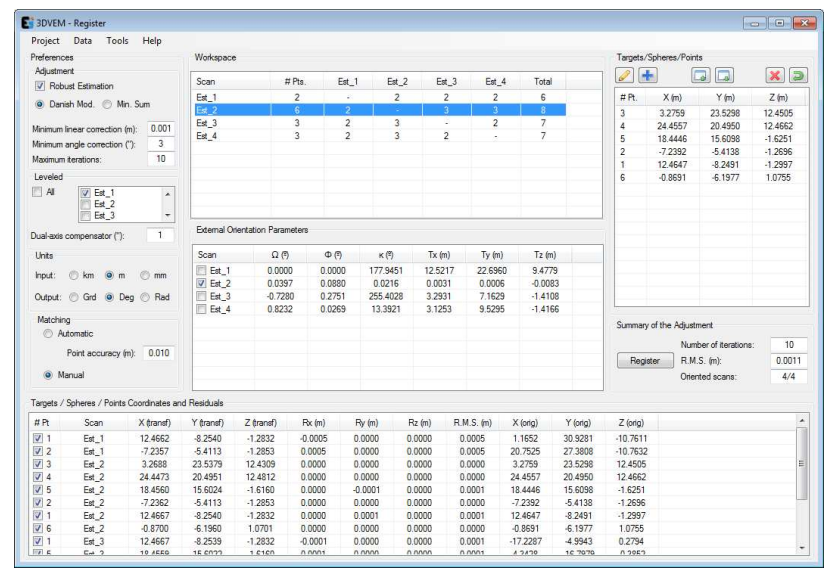

Figure 3. Main window of 3DVEM - Register software

3DVEM - Register is software specifically designed to solve any complex laser scanning registration process with maximum precision. The software always yields the best achieved solution.

Figure 4 shows a top view of several Light Detection And Ranging (LiDAR) point clouds captured with a terrestrial laser scanner system before and after registration. The survey of the Mirage F-1 aircraft was undertaken at the Universitat Politècnica de València.

A summary of the most important 3DVEM - Register features are presented next:

- $\quad$ Flexible and easy to use registration adjustment: cloud to cloud, cloud to surface or surface to surface.

- Full export detailed report with estimates and residuals of the adjustment. 
- $\quad$ Least squares adjustment and robust estimation (Danish Modified and Minimum Sum method). Robust estimation is recommended to detect gross errors in the input data.

- There is a chance to carry out either automatic mode (automatic detection homologous points), manual mode (points with known IDs) or a combination of both.

- Import/export in various file formats: ASC, TXT, PTS, PTX, LAS, LAZ, FLS, FWS, OBJ, WRL and DXF.

- Import/Export of targets/spheres/points in the following formats: PTS, ASC, TXT, CSV, XLS and DXF.

- Export results to VRML and DXF files. The CAD file is structured in different layers: point clouds, positions, and targets.

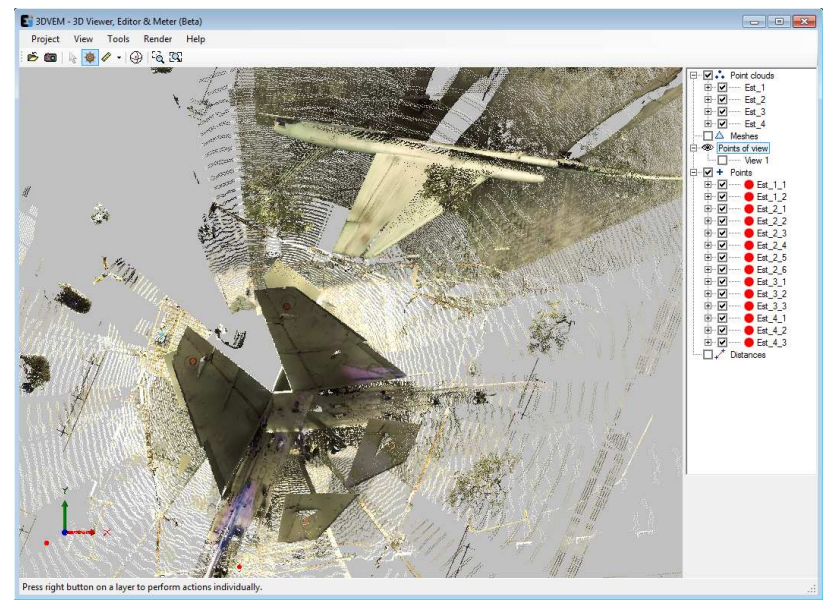

(a)

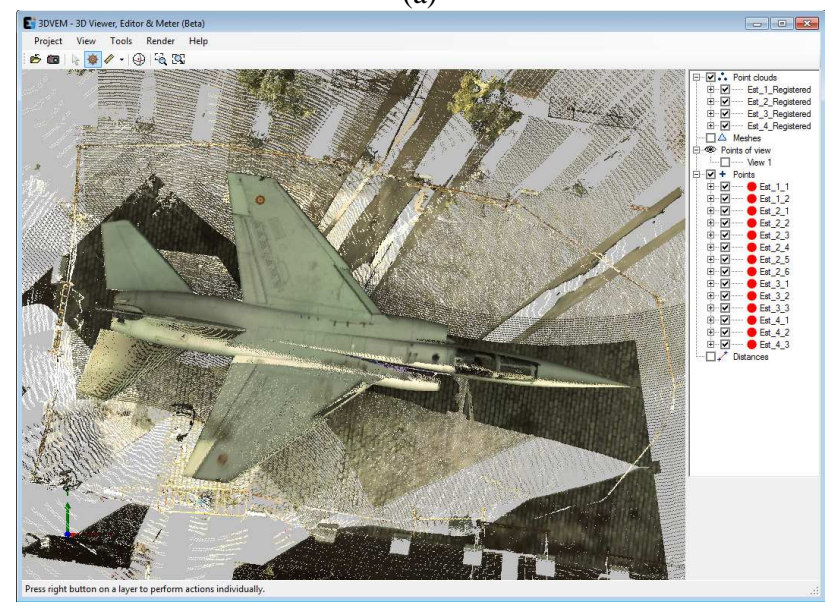

(b)

Figure 4. Top view of the colour laser scanning point clouds acquired from four stations: a) raw (unregistered); b) registered

\section{3DVEM - LIVE TO ENHANCE INTERACTION, ANIMATION AND DISSEMINATION}

3DVEM - Live software is a low cost powerful enhanced version of 3DVEM - 3D Viewer, Editor \& Meter software. Tools are added to allow users to develop multimedia products based on external imagery and inner animation tools. It makes 3DVEM - Live an optimum software tool that eases cultural heritage research, preservation, dissemination and last but not least entertainment. It is a module designed for information users such as archaeologists, architects, conservator, art restorers and museum staff, among others, to enrich virtual models with their knowledge.

3DVEM - Live allows users to enrich themselves photorealistic 3D models with their own images through landmarks. A landmark can be used to input related information in the form of text and/or image. Once created, the landmark may be deleted or edited. Figure 5 shows the program 3DVEM - Live in presentation mode (full screen) making use of rock art shelter Cantos de Visera II (Fig. 1) with added landmarks.

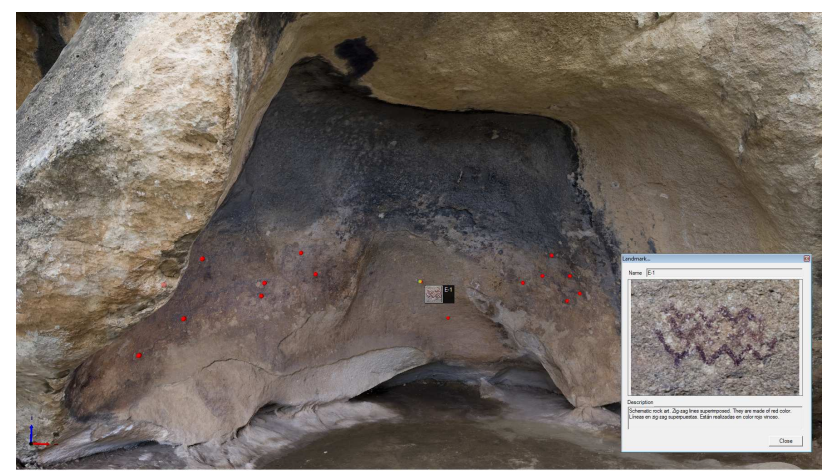

Figure 5. Presentation mode of 3DVEM - Live software displaying landmarks

3DVEM - Live also integrated the chance to create virtual animations in both mono and stereo modes. Application users are able to generate as many virtual animations as they wish with/without rendering. Animations can be easily created in two steps through viewpoints. The program allows you to show or hide the camera position and the camera path (Figure 6). The animations can be displayed in real time in presentation mode or recorded in AVI format at different resolutions (Figure 7).

3DVEM - Live can also be operated in closed mode where some features are locked to avoid misuses, for example, in museums where visitors can navigate and interact freely with the $3 \mathrm{D}$ data sets, namely photorealistic 3D model.

Some of the most important features 3DVEM - Live software are presented next:

- Anaglyph rendering. Figure 8 shows the stereoscopic rendering of the main door of the Palace of Marquis of Dos Aguas (currently National Ceramics Museum) in Valencia (Spain).

- Create and save virtual animations and video recording.

- Insert landmarks with related information (image and text). Closed mode to facilitate access non-expert users.

- Ability to change camera settings.

The parallax-free stereoscopic visualisation enhances the chances of communication and interpretation of the monuments, sites, objects and artifacts. Fig. 8 might be a good example of delicate ornamentation on a façade. The user can select optionally the preferred rendering mode to display the animations and/or snapshots on the screen projector, panel or multimedia display. 


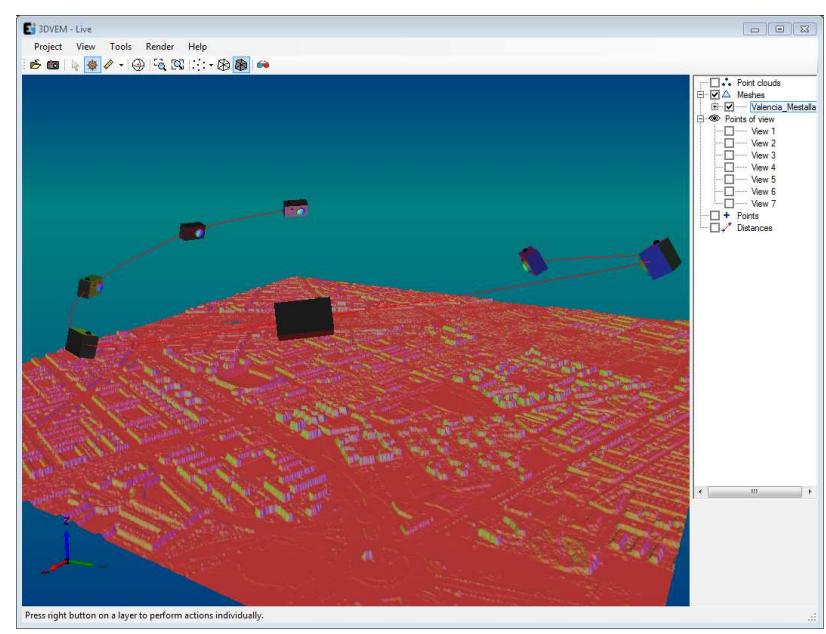

Figure 6. 3DVEM - Live software displaying the camera path to create the animation

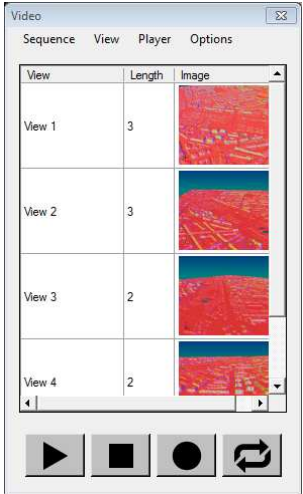

Figure 7. Video window

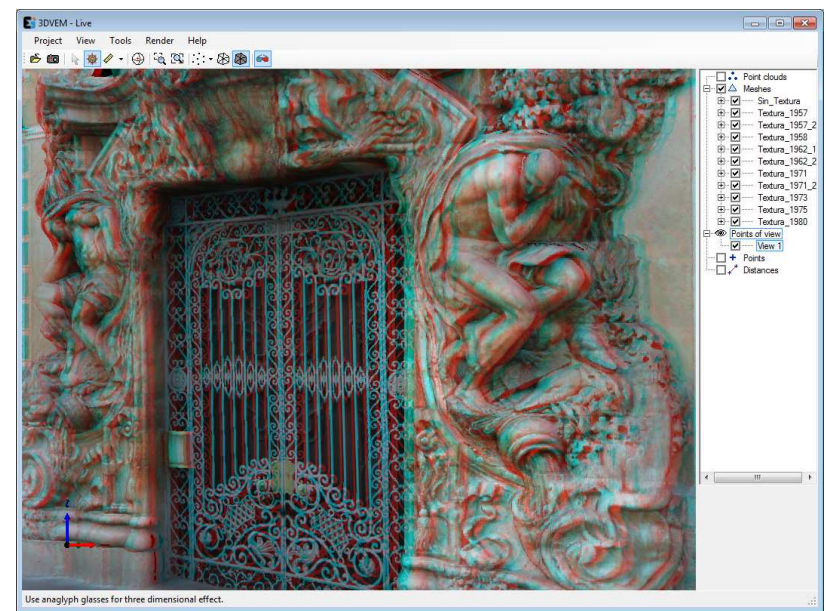

Figure 8. Stereoscopic rendering of a photorealistic 3D model

\section{CONCLUSIONS}

This paper presented three user friendly 3DVEM software modules thought to enhance the performance of both information users and information providers when dealing either with 3D point clouds or with photorealistic 3D models. The input data might have been acquired by any surveying device or optical 3D measuring system.
The registration software 3DVEM - Register targets information providers really concerned with the quality of the processing outputs. In addition, automation and variety of 3D outputs aims to support multidisciplinary teams dealing with point clouds, 3D models, computer graphics, computer vision, laser scanning, surveying and photogrammetry.

Depending on the user's needs, the chance to select between 3DVEM - 3D Viewer, Editor \& Meter or 3DVEM - Live is up to the costumer. The former is free while the latter is considered low cost software. The easiness of handling, labelling, inserting pictures, animating sequences and rendering makes a difference.

Further 3DVEM software modules will be launched in the near future to improve the performance of both image-based and range-based solutions existing in the market.

\section{REFERENCES}

Autodesk Inc., 2013a. Products Overview: Autodesk 3ds Max. http://www.autodesk.com/products/autodesk-3ds-max/overview (10 Jul. 2013).

Autodesk Inc., 2013b. Products Overview: Autodesk Maya. http://www.autodesk.com/products/autodesk-maya/overview (10 Jul. 2013).

Blais, F., 2004. Review of 20 years of range sensor development. Journal of Electronic Imaging, 13(1), pp. 231240.

Blender Foundation, 2012. Features. http://www.blender.org/features-gallery/features/ (10 Jul. 2013).

Bryan, P. G., Barber, D. M. and Mills, J. P., 2003. Towards a standard specification for terrestrial laser scanning in cultural heritage - One year on. International Archives of Photogrammetry and Remote Sensing, XXth ISPRS Congress, 12-23 July 2004 Istanbul, Turkey, Commission V, pp. 10301036.

Cignoni, P., Callieri, M., Corsini, M., Dellepiane, M., Ganovelli, F., and Ranzuglia, G., 2008. MeshLab: an OpenSource Mesh Processing Tool. Eurographics Italian Chapter Conference, pp. 129-136.

GIFLE, 2013. Photogrammetry \& Laser Scanner Research Group. Services. Software development: 3DVEM, 3DVEM Register. http://gifle.webs.upv.es/Services.php (28 Jun. 2013).

Lerma, J.L., Navarro, S., Cabrelles, M., Villaverde, V., 2010. Terrestrial laser scanning and close range photogrammetry for 3D archaeological documentation: the Upper Palaeolithic Cave of Parpalló as a case study. Journal of Archaeological Science, 37 (3), pp. 499-507.

Lerma, J.L., Cabrelles, M., 2013. Documentación 3D y difusión multimedia del yacimiento de Arte Rupestre Cantos de Visera II. YAKKA. Revista de Estudios Yeclanos, 19 (2011-2012), pp. 275-280.

Letellier, R., Schmid, W., LeBlanc, F., 2007. Recording, documentation, and information management for the conservation of heritage places. Volume I: Guiding Principles. The Getty Conservation Institute, Los Angeles. 
MeshLab, 2012. Meshlab. Visual Computing Lab - ISTI - CNR http://meshlab.sourceforge.net/ (10 Jul. 2013).

Ruíz Molina, L., 2013. 100 años de arte rupestre. Cantos de Visera. Monte Arabí Yecla (Murcia) YAKKA. Revista de Estudios Yeclanos, 19 (2011-2012), pp. 13-289.

\section{ACKNOWLEDGEMENTS}

The authors gratefully acknowledge the support from the Spanish Ministry of Science and Innovation to the project HAR2010-18620. 